\title{
Towards Designing Effective Governance Regimes for Smart City Initiatives: The Case of the City of Duisburg
}

\author{
Peder Bergan \\ University of Duisburg-Essen \\ peder.bergan@uni-due.de
}

Frederik Ahlemann

University of Duisburg-Essen

frederik.ahlemann@uni-due.de

\author{
Anne-Marie Mölders \\ University of Duisburg-Essen \\ anne-marie.moelders@uni- \\ due.de \\ Stefan Decker \\ University of Duisburg-Essen \\ $\underline{\text { stefan.decker@uni-due.de }}$
}

\author{
Kevin Rehring \\ University of Duisburg-Essen \\ $\underline{\text { kevin.rehring@uni-due.de }}$ \\ Stefan Reining \\ University of Duisburg-Essen \\ stefan.reining@uni-due.de
}

\begin{abstract}
Smart cities are characterized by heterogenic stakeholders, many layers of authorities, complex decision-making processes, and competing objectives. As a result, they require a sophisticated and wellplanned governance regime. We describe the development and design of a governance regime which is grounded on IS principles as well as the resulting governance structure in a medium-sized city in Europe. Using the action design research approach, we designed, implemented, and revised in multiple iterations an ensemble artifact consisting of the governance structures and processes for a smart city initiative. Our empirical observations highlight challenges of coordination, communication, and innovation in this smart city and report on how we implemented and adjusted the governance regime accordingly. Our results are a first step towards general recommendations for the design and implementation of Smart City governance regimes in medium-sized cities.
\end{abstract}

\section{Introduction}

Cities are considered key elements for the world's future well-being. The number of citizens living in cities is increasing rapidly, and the resulting high concentration of people brings challenges such as increased traffic jams, waste disposal, and greenhouse gas emissions [10]. In response to these challenges, many cities have become involved in smart city (SC) initiatives, making use of and combining innovative information and communication technologies (ICT) [9]. Europe, partially due to the European Commission's engagement in this respect, will have the largest number of SC initiatives globally [13]. Since the number of
European SC initiatives is increasing and they struggle to implement SC initiatives due to technical, managerial, governance, and financial challenges $[1,25$, 26], we maintain that it is crucial to understand the underlying processes to enable effective implementations.

SC initiatives share some important characteristics with digital transformation (DT) initiatives in firms [25]. These characteristics include the use of ICT-based infrastructures and service environments [3], as well as a plethora of different stakeholders affected by the transformation, all of which require excellent management [9]. Moreover, stakeholders' expectations are changing, with citizens expecting more convenience, online information, and the asynchronous handling of data [11]. Modern technology usage, which accelerates the speed of change, requires shorter planning horizons, increasing the management complexity further [9].

There are also some notable differences between SC initiatives and DT in enterprises. Smart cities comprise a substantial amount of complexity compared to ordinary IT projects [26]: the public sector often consists of a variety of multi-level authority structures, which could negatively impact the speed and efficiency of SC implementation [6]. The decision-making processes' complexity arises from networks of multiple, distinct legal entities [9] such as enterprises, schools, non-governmental institutions, local governments, transport companies, etc. [30], all of which have different cultures, IS capabilities, and goals. Orchestrating such digitization initiatives in a public environment requires comprehensive SC governance [9, 30]. Medium-sized cities are of particular interest in this regard because they comprise the majority of European cities and may have lower organizing capacity than large cities. 
Previous literature has called for research on SC governance patterns' efficacy and efficiency and identified the scarcity of literature linking concepts from the field of information systems (IS) to the SC concept $[9,38]$. Researchers have also called for an examination of governance in smart cities as one that requires an alignment between the organizational structure, IT elements, citizens, and the governance processes [9, 21]. Addressing this research gap, we aim to increase the understanding of how to design governance regimes and optimize their alignment for smart cities by answering the following research question (RQ): How can a smart city governance regime be designed and implemented in a medium-sized European city?

As such, we provide a primarily descriptive approach for the design of an SC governance model in the context of an SC initiative in a medium-sized European city. This is meant to be a starting point for further prescriptive research on SC governance's effective implementation in medium-sized European cities. We follow the action design research (ADR) approach, defined by Sein et al. [32], because it reflects the way we took part in designing the initiative and since ADR (see 3.1) is suited to guide the creation of artifacts like the described SC initiative from the very beginning. Our approach is similar to the ADR model proposed by Mullarkey and Hevner [24], who extended Sein et al.'s [32] ADR model by presenting the cycles as a series of discrete and well-defined interventions and by including the creation of cycle-specific artifacts [24]. Thus, the paper is structured as follows: Section 2 introduces the concepts related to a SC and its governance. Section 3 describes and justifies our ADR application and provides an overview of our case city. Section 4 describes the development process for the SC governance regime in our case city (4.1) as well as the elements of the resulting governance model (4.2). Section 5 discusses the generalizability of our results and mentions limitations and opportunities for further research.

\section{Theoretical foundations of smart city governance}

A growing body of literature has investigated the SC concept. However, the meaning of the term 'smart city' is multi-faceted, because its definitions refer to diverse characteristics related to citizens, IT, infrastructure, mobility, the environment, the economy, and more [2]. This paper will use the definition by Caragliu et al. [7] because it considers SCs' human and technological dimension, reflects a modern conception of SC in research, and has become increasingly common. According to this definition, a city is smart when "investments in human and social capital and traditional (transport) and modern (ICT) communication infrastructure fuel sustainable economic growth and a high quality of life, with a wise management of natural resources, through participatory governance" [7].

The integration of private and public sector organizations is a crucial aspect contributing to SC governance's complexities. The public sector has many layers of authority, which may lead to delayed decisionmaking [34]. Owing to public sector organizations' political nature, long-term planning may be difficult, because political cycles can cause management turnover and corresponding changes of priorities [34]. This blend of different elements and organizations leads to "diverse stakeholders, high levels of interdependence, competing objectives and values, and social and political complexity" [9], indicating the unique challenge involved in endeavoring to establish a matching governance regime. This blend is also strongly related to technology, e.g. the variety of IS, networks, security policies, etc. and the frequent challenges of having to integrate various organizations' silo applications.

Organizational governance is commonly defined as "the determination of the broad uses to which organizational resources will be deployed and the resolution of conflicts among the myriad participants in organizations" [12]. Applied to SCs, it addresses the need for managing its resources efficiently as well as define decision rights and responsibilities. Since ICT is a key resource in SCs, the underlying governance should likewise "encourage desirable behavior in using IT" [42]. These can be deployed by defining "a combination of processes, structures and relational mechanisms" [6]. Structures include for instance committees or councils, while processes describe the monitoring of decisionmaking, e.g. through management reporting and automated procedures [6]. Owing to the nature of the challenges listed in the previous section, we emphasize the organizational rather than IT aspects of governance for SC initiatives, although both remain integral.

Importantly, the term 'smart city governance', as used in existing SC literature, often refers to government processes of smart cities. E.g., Meijer and Bolívar present "four ideal-typical conceptualizations of smart city governance", ordered along "a scale ranking from institutional conservation (traditional governance of a smart city) to institutional transformation (smart urban governance)" [22]. Similarly, Castelnovo et al. provide a framework for assessing the performance of SC governance in terms of "changes in city government systems" [8] generated by ICT-enabled innovations. These uses of the term 'smart city governance' refer to the area of smart governance, which forms only one of various dimensions of the smart city concept [20]. In 
contrast, 'SC governance', as used in this paper, refers to the governance of SC initiatives, that is, to the organizational structures and processes involved in SC transformations. Research has shown that there is no uniform model for the governance of SC initiatives. Governance models implemented by existing initiatives range from participatory to hierarchical [1], depending on the centrality of decision-making processes and the inclusion of various stakeholder groups into these processes. Moreover, SC governance regimes highly vary in their degree of formality [1]. Overall, the governance of SC initiatives - especially in terms of detailed descriptions of organizational structures and processes - is an aspect that is still underexplored in existing SC literature.

\section{Research design}

This section introduces the applied action design research (ADR) model and the case city. The artifacts' design is grounded in relevant IS principles and is transferrable to SC initiatives due to the previously described core similarities between organizational governance and SC governance.

\subsection{An action design research approach}

ADR consists of a combination of design research (DR) and action research (AR) [32]. While DR "seeks to develop prescriptive design knowledge through building and evaluating innovative IT artifacts intended to solve an identified class of problems" [32], AR aims to investigate changes' consequences within a specific application domain directly [25, 31]. Consequently, ADR can generate "prescriptive design knowledge through building and evaluating ensemble IT artifacts in an organizational setting" [32] to solve a practical problem, which is in line with what was asked of us in the case city. ADR is suitable for addressing our RQ, because, in accordance with the ADR definition mentioned above, the structures and processes developed in the course of the SC initiative constitute an ensemble artifact. Moreover, the artifacts' development took place within a concrete application context and aimed to solve a practical problem.

Our research process's stages are similar to the ADR model by Mullarkey and Hevner [24], who adapted and complemented Sein et al.'s [32] initial approach. Mullarkey and Hevner's [24] model contains four overarching stages: diagnosis, design, implementation, and evolution. In the diagnosis stage, we identified the practical problem, which generally comprised the need for the city of Duisburg's digital transformation, and several related sub-goals, after which we started gathering the most relevant theoretical explanations. In the design stage, we identified and conceptualized the proposed artifact design and drafted the first version of the so-called masterplan, which defined the governance's underlying requirements and a basic blueprint of the SC initiative. In the implementation phase, we realized the governance regime by creating processes and structures in practice, constantly reevaluating these. In the evolution stage, we considered the changes in the problem environment, which related to the stakeholders' increasing heterogeneity and the artifact's evolvement. As in Mullarkey and Hevner's model, each stage consisted of multiple iterations of a cycle of problem formulation/action planning, artifact creation, evaluation, reflection, and formalization of learning [23]. During the process, two types of artifacts were created: organizational structures and governance processes, including their decision rights and accountabilities.

We analyzed collected data by adhering to the principle of theory-ingrained artifacts [32], ideas and concepts for the SC initiative were based on high-level IS research that was translated into the SC governance context. In addition, we consulted market analyses and seminal academic papers from the field of SC research. During the governance regime's development process, we collected data on the city's environment and the stakeholders through 10 interviews and 6 workshops. We cooperated with the city officials to identify relevant types and sources of data, gathered the initial datasets, and helped assess it as a foundation for decisionmaking, which is crucial in SC [31]. These data streams were divided into human (stakeholder interviews, surveys, workshops, etc.) and technical-oriented streams (sensors, big data, etc.). A detailed description of data analysis techniques is presented in chapter 4.1.

\subsection{Case: Smart City Duisburg}

The investigated case is the SC initiative of the city of Duisburg, located in the western part of Germany. Duisburg has approximately 500,000 inhabitants, making it a representative example of a medium to large-scale European city [27]. This type of city now constitutes the "most important class of cities in Europe in demographic terms" [14]. Duisburg faces challenges typical for medium-sized cities including less resources, funding and organizing capacity compared to large cities [14] and exhibits several characteristics that are representative of medium-sized cities including a good access to various transportation networks [18]. In the context of the SC initiative, the lord mayor and his subordinates had a political mandate to explore the digital transformation of Duisburg, exploit the advantages, but avoid the challenges that arise with such 
a transformation. Like most smart cities, the city of Duisburg also clusters the most relevant topics into domains or topic areas, divided into smart economy, smart mobility, smart living, smart education, broadband, smart infrastructure, and e-government.

For the transformation to occur as smoothly as possible, close collaboration and sharing of knowledge was required between the city, research institutions, and local and city-owned companies. In this regard we were involved from the very beginning of the initiative and received privileged access to relevant data. In the course of the initiative, the different parties together developed a masterplan as well as the standards and principles used to establish the initiative. We started our research work in the case city with a thorough diagnosis which we outline in the following section, structured in line with the ADR cycles by Mullarkey and Hevner [24].

\section{Results}

4.1. Development process of governance regime 4.1.1 Diagnosis of needs for the smart city transformation. The ADR diagnosis phase began in July 2017, lasted about six months and was planned in close interaction with the city's IT executive. In order to discuss and structure expectations and perceived challenges about the SC initiative, we conducted a series of kickoff meetings and, later, formal regular meetings with various city officials including the deputy major, the head of IT management, the deputy IT manager, the head of digitization department, as well as the IT director. We took part in and offered workshops about the fundamentals of digitization, requirements of digital strategies for cities, generation of SC-related project ideas, and funding of SC initiatives. Following the guidelines by Michalko [23], we used the techniques brainstorming, mind mapping, idea box, and future scenarios to enable participation and collaboration among the stakeholders.

Applying these methods, we found that the city's major problem was to find ways to orchestrate and coordinate the various SC stakeholders - in particular regarding structured strategic decision making and operational control of the projects to come. Therefore, in workshops with managers from the partners we collaboratively analyzed the problem and collected requirements and initial approaches to overcome some of the challenges. This data collection and analysis allowed the crystallization of the overarching vision of the SC with its superordinate goal of interconnecting its citizens, the economy, different societal groups, the politics, and the administration by means of modern ICT. The SC's main goal was defined as increasing the residents' quality of life, as well as the city's economic attractiveness and sustainability. Researchers and city officials agreed that a governance regime should serve these objectives by setting up a plain and scalable governance structure to ensure that the predefined goals of the initiative could be effectively reached while balancing innovation and risk. In addition, researchers and city officials agreed upon a set of fundamental principles that should guide both the design of the later governance regime and decision-making processes. For instance, one principle said that the SC initiative needed to be benefit-oriented, avoiding solutions that are neither economically nor otherwise useful.

We also developed a mind map of the key players that the SC initiative would affect, because understanding who these stakeholders were, their needs, and their influence were of the utmost importance in the SC governance development process [4]. The stakeholders were sorted into categories, which included the city of Duisburg, the university, business, society, finance, culture, tourism, health, and sports. An internal review identified a subset of stakeholders crucial for the SC initiative's governance based on their function and influence. We also derived attractive benefits for the citizens and companies in order to increase acceptance and use by all the stakeholders, which remains a challenge for many SCs [33]. In SCs, benefits management is an important discipline to increase the likelihood of producing clear and measurable benefits [41]. Benefits management methods "relate outcomes to business changes needed, address the context issues to some degree and certainly engage the business managers in the process more effectively than most IS/IT methods" [39]. Ward, Taylor, and Bond [40] suggest planning and assigning "well-defined responsibilities" for business changes. Accordingly, we systematically divided the overall vision of the SC Duisburg into several sub-goals that would prioritize and operationalize the benefits according to, for example, cost-benefit considerations. We designed and created a SC monitoring of performance indicators across several domains of activity, including the number of ideas submitted by citizens, running projects, social media mentions, and the amount of research funding granted.

4.1.2 Design of principles, communication, and partner management. In the design phase, we took the results from the previous phase as design requirements for the development of the masterplan document. This document was supposed to describe the overall organizational structure, processes and the governance regime for the SC initiative. It was meant to serve as a blueprint for later implementation and was planned to be approved by the city council. We developed and revised the masterplan as a superordinate artifact. In 
several iterations, we created the artifact's components which included (1) the decision-making entities, (2) their accountabilities on the project, portfolio, and program level, and (3) a high-level depiction of the planning and steering processes required. Furthermore, we designed the domains which serve as a structure for the overall regime and activities. This procedure was based on insights from DT research showing that highperforming enterprises use a variety of ways to generate value from IT, including a business strategy as well as the measurement and management of the resources spent [42]. The completed masterplan therefore contains a detailed milestone plan providing a schedule of the necessary and verifiable events. These activities' effective management requires an architectural design promoting the development of an environment that supports and creates synergies among SC projects [16]. As a result, we specified underlying architecture requirements which served as a foundation for SC architecture principles. These requirements were not only technological, but also organizational and included requirements for, among others, data exchange, reusability, and the design of a shared SC platform.

Frequent and thorough communication routines can significantly increase end-users' understanding and use of IS (which many of the SC projects in the case city resulted in) but these routines require planning [5]. We therefore created a communication policy that considers both the intended recipient and different media. Media include the initiative's web site, social media, postal mail, and press and mail correspondence. The policy also contains information about the organizational structure of the communication, including the roles and responsibilities. We subsequently developed a partner concept with an approach to acquire and manage the SC initiative's potential partners that is closely related to the communication policy. For instance, the partner concept specifies that whenever existing partners present the SC initiative, they should encourage the involvement of more stakeholders and invite potential partners to discuss this in detail. Recruited partners are managed in terms of including them in each board and group's activities, by ensuring that they adhere to the architecture principles and communication policy. Potential partners should be requested to undertake one or more SC projects in cooperation with the existing partners. This helps to assess such partners, teach them about the SC governance regime and architecture, and stimulate projects.

On the individual level, we also designed processes for participative workshops that allow citizens to provide feedback and new ideas for projects. In such workshops, experienced researchers and practitioners guide interested citizens and provide valuable ideas to advance an SC initiative. Additionally, we conducted interviews with 8 managers and experts of local companies to better understand their practical perspectives on SC transformation. In a stage-gate process, the initiative's program management (including this paper's authors) assessed the new ideas, made decisions about them, and - when necessary modified them. This process not only led to an increased acceptance level, but also served to generate innovative ideas that reflected the public's opinion [15].

4.1.3 Implementation, evaluation, and adoption of governance processes. The implementation phase, beginning in July 2018, consisted mainly of the previously established masterplan's realization by the city and partners with our guidance. The phase was launched via an official kick-off and networking event organized by the city. This event served to ensure and display the official commitment by the city administration and involvement of key partners, which is a fundamental factor in SC development [25], inform stakeholders about the plan, and request participation.

The program management activities proposed in the masterplan were also further specified, with the city requesting more guidance for the program management, due to the large number of stakeholders and the high degree of interdisciplinarity. Since SC governance comprises multiple projects, we relied on Tiemeyer's [36] recommendations for multi-project management. In a separate intermediary design cycle, we developed new guidelines to define the processes for the initiative's project/portfolio management. Defining these processes revealed tensions about partners' information reporting to the program management. Partners wanted to protect confidential information and minimize reporting effort, but the initiative could improve cooperation and resource use by sharing information. In response, we created a working group at the program level consisting of mid- and low-level partner representatives and researchers, aiming to exchange information about ideas, funding, potential partners, etc. The program management and this working group distributed available capacities among the projects so as to allow the latter to efficiently use resources in a balanced way over time, corresponding to each project's stakeholders and priority [36]. Where possible, initiatives with a short planning horizon are favored to allow them to react quickly to new technological developments and to minimize the risks. This approach necessitates continuous, rolling planning by the program and portfolio levels.

During the implementation phase, the previously established boards began their operations. One of the main organizational structures, the innovation center, was also founded and set up as a virtual organization. This set-up included a letter of intent that the initial 


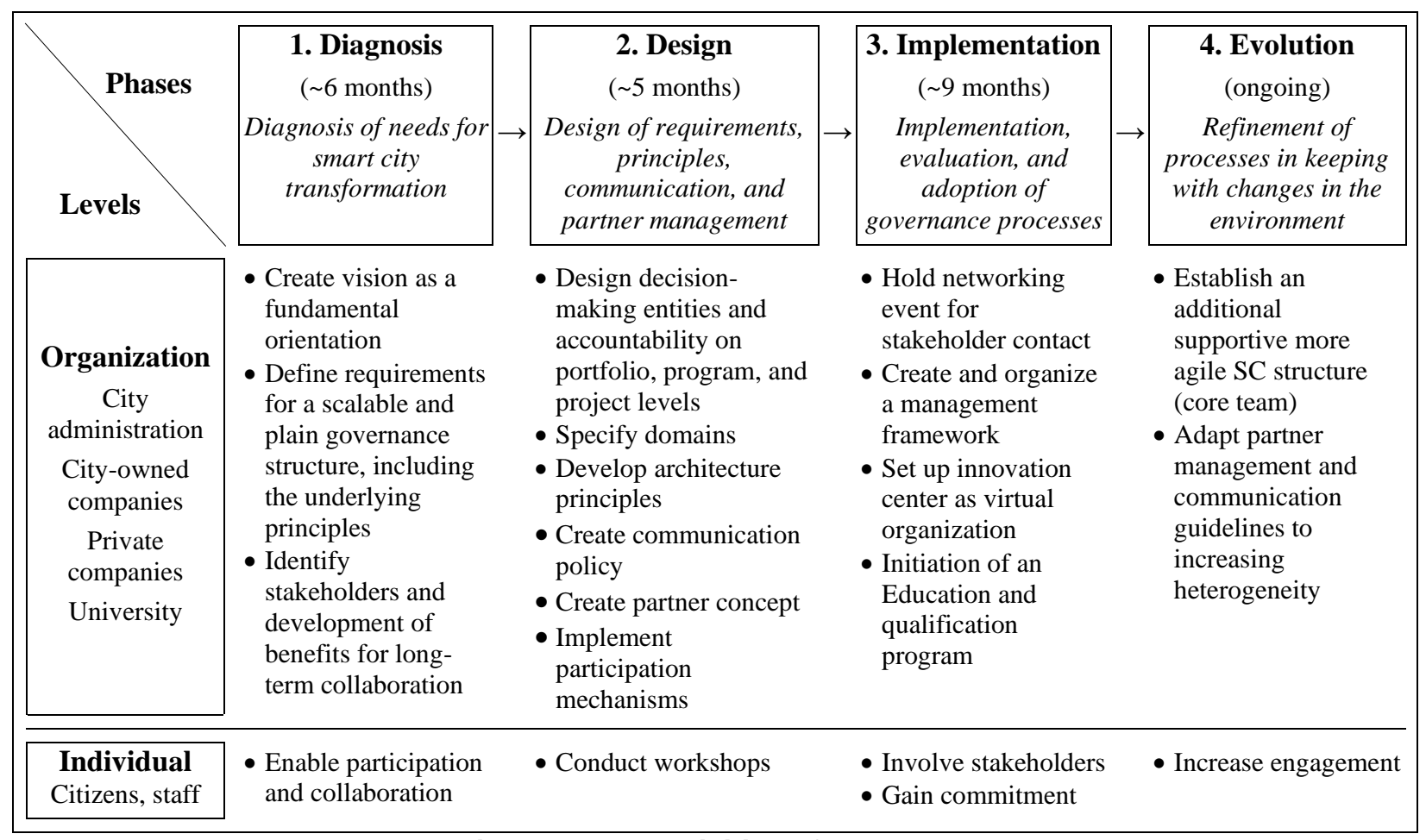

Figure 1: Key activities of each cycle

partners signed and that enabled the official set-up, the appointment of a leader and small staff, a kick-off event, and efforts to acquire more partners. The initiative's supporter alliance comprises the city of Duisburg, (municipal) companies, social institutions, and research institutions.

Given the high degree of interdisciplinarity and the great digital expertise required for an SC transformation, we suggested establishing a professional education and qualification program of different expertise levels and focus areas. Using workshops with the initiative's partners, we elicited needs and defined the curriculum and modalities. On the organizational level, an education and qualification program could improve companies' understanding of DT, including the relevant risks and opportunities. An education and qualification program could also connect different stakeholders, helping them to create networks through which they can discuss practical cases related to the SC initiative. Such a program would therefore not only improve skills but would also help organizational cultures adapt to an SC transformation's requirements.

The implementation phase also included the completion of the previously planned workshops based on the SC domains, which went hand in hand with the benefits management's implementation. The ideas the program management gathered from the workshops and partner meetings were sorted by their main anticipated benefits and ranked according to a small set of qualitative evaluation criteria. After an idea had been selected, the program management conducted a more detailed analysis during which more (and quantitative) benefit categories were assessed. The program management team analyzes the project proposals' costs, benefits, dependencies, and responsibilities. Based on this evaluation, the program management presents the highest scoring ideas as a "shortlist" to the steering committee, who makes a preliminary decision to initiate a pre-project (or not). The program management then organizes a business and technical analysis of each idea, generally conducted by experts in the partner organizations, resulting in a quantified assessment of the extent to which the citizens would benefit from the proposed project, as well as of the costs and technical and organizational challenges. At the end of the implementation phase, the masterplan, including its components, had been largely realized. The process involved many smaller iterations during the implementation and design cycle, due to the SC initiative's and its stakeholders' agile management and interdisciplinarity.

4.1.4 Refinement of processes in keeping with changes in the environment. In the evolution phase, artifacts are developed further and refined to adapt them to changes in the problem environment [24]. In the case of the SC initiative, the relevant environmental changes mainly comprised the increase in the number of partners, since an increasing number of citizens and companies became aware of and interested in 


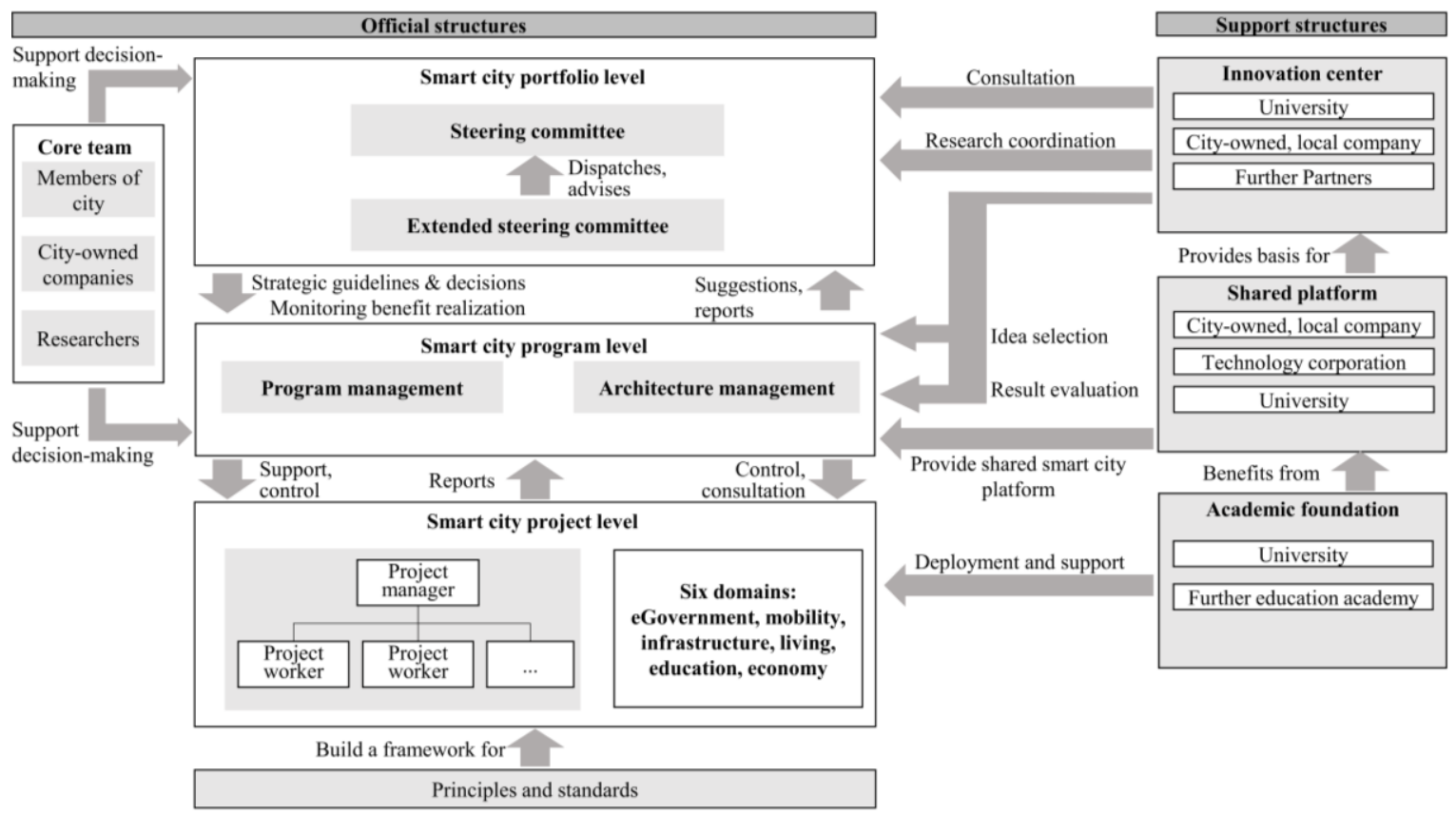

Figure 2: Main organizational structures and activities of the SC initiative

influencing the initiative. Since the steering committee only met quarterly, the strategic decision-making processes were too slow to react to changes in the problem environment. Consequently, establishing a separate core team was suggested by several partners, which resulted in a change in the overall governance structure. The core team operates mostly independently and is partially positioned between the program management and the steering committee and is allowed to make decisions of medium importance. This team follows a more agile approach regarding meetings, management, and decision-making. It comprises city councilors, city-owned company managers, domain experts, and researchers.

Over time, we also observed that the actors lacked the necessary in-house capabilities to properly conduct certain SC activities (e.g. IoT software development) and that incumbent technology providers did not always offer suitable solutions for the SC domain. Therefore, and given the prospect of synergetic learning effects, we extended the partner concept with explicit benefits and support for startups: corporations would help startups improve their products and performance, while the startups would support innovation [17].

Furthermore, the stakeholders' increasing heterogeneity required a change to and refinement of the communication policy. Outside companies had hitherto approached each partner individually, which resulted in incoherencies and became a point of tension. To resolve this, the program management improved the sharing of information about projects and set up joint meetings to ensure a more coherent communication. The program management and innovation center staff refined the partner management, including a process where partners nominate new ones, aiming to increase engagement. The partners are assigned a bronze, silver, gold, or platinum status, depending on the resources they invest in implemented SC projects. Each partner level also includes increasingly strict requirements about adhering to SC architecture principles. The partners' access to the organizational structures and their influence on the governance processes increased when the partner level increased.

\subsection{Governance model}

The ensemble artifact that resulted from the ADR activities described in the previous section comprised the organizational structures, key processes, roles, vision, goals, and domains. Figure 2 provides an overview of the organizational structures and key activities. In what follows, some key elements of this artifact are described in more detail.

On the portfolio level, the steering committee, which makes decisions about projects' strategic alignment, prioritization, and budgeting, chiefly leads the initiative. This ensures that the initiative is centrally governed and politically accountable. The extended steering committee advises the steering committee and generates new ideas for the SC initiative. In addition, the extended steering committee receives required information about the projects and dispatches information from its members to the steering committee. The program level 
Table 1: Responsibility assignment matrix of the SC governance

\begin{tabular}{|c|c|c|c|c|c|c|c|c|c|}
\hline \multicolumn{2}{|r|}{ Process description } & \multicolumn{8}{|c|}{ Organizational structure } \\
\hline Process & Name & SC & ESC & $\mathbf{P M}$ & $\mathbf{E}$ & IC & $\mathbf{A M}$ & PL & $\mathbf{P}$ \\
\hline A1 & Stage-gate Process & & & & & & & & \\
\hline A1-P1 & Management of ideas and alignment with initiative goals & A & $\mathrm{C}$ & $\mathrm{R}$ & $\mathrm{I}$ & $\mathrm{C}$ & $\mathrm{C}$ & $\mathrm{I}$ & $\mathrm{I}$ \\
\hline A1-P2 & Identification, analysis, and management of stakeholders & A & $\mathrm{C}$ & $\mathrm{R}$ & $\mathrm{I}$ & $\mathrm{C}$ & $\mathrm{C}$ & $\mathrm{I}$ & I \\
\hline A2 & Portfolio Management & & & & & & & & \\
\hline A2-P1 & Creation of the project portfolio & A & $\mathrm{C}$ & $\mathrm{R}$ & I & $\mathrm{C}$ & $\mathrm{C}$ & I & I \\
\hline A2-P2 & Control and regulation of the portfolio & A & $\mathrm{C}$ & $\mathrm{R}$ & $\mathrm{I}$ & $\mathrm{C}$ & $\mathrm{C}$ & $\mathrm{I}$ & I \\
\hline $\mathbf{A 3}$ & Communication & & & & & & & & \\
\hline A3-P1 & Creation, refinement, and monitoring of the communication policy & I & $\mathrm{I}$ & A & I & $\mathrm{C}$ & $\mathrm{C}$ & I & $\mathrm{I}$ \\
\hline A4 & Architecture management & & & & & & & & \\
\hline A4-P1 & Identification, orchestration, and monitoring of the architectural elements & I & $\mathrm{I}$ & $\mathrm{C}$ & $\mathrm{I}$ & I & A & $\mathrm{C}$ & $\mathrm{C}$ \\
\hline A5 & Partner management & & & & & & & & \\
\hline A5-P1 & Establishment and maintenance of the partner concept & I & I & A & I & $\mathrm{R}$ & $\mathrm{C}$ & I & $\mathrm{I}$ \\
\hline A5-P2 & Establishment and maintenance of start-up support structure & $\mathrm{I}$ & $\mathrm{I}$ & A & I & $\mathrm{R}$ & $\mathrm{C}$ & $\mathrm{I}$ & $\mathrm{I}$ \\
\hline A6 & Monitor governance structures and processes & & & & & & & & \\
\hline A6-P1 & Adjustment of the organizational structure & A & $\mathrm{C}$ & $\mathrm{C}$ & I & $\mathrm{C}$ & $\mathrm{C}$ & I & I \\
\hline A6-P2 & Monitoring of the initiative and identification of the optimization potentials & $\mathrm{R}$ & A & I & I & $\mathrm{C}$ & $\mathrm{C}$ & $\mathrm{I}$ & I \\
\hline
\end{tabular}

implements the steering committee's requirements and is responsible for the meetings' preparation and followup to enable smooth and efficient decision-making processes. The program management collects, specifies, and reviews project ideas, all of which enable the steering committee to make decisions. Subsequently, according to the steering committee's requirements, the program management monitors and manages the set of projects in the different SC domains, by eliciting and analyzing project reports, using more in-depth project health checks, providing advice about stakeholders, etc. Therefore, to support the initiative's overarching goals the program management uses a portfolio of all ideas and projects with classifications and assessments such as SC domain, benefits, risks, effort, available resources, etc. [19]. The program level is also responsible for the superordinate topics' coordination, which includes the regulatory aspects, data security, templates, project auditing, and quality control. The operative project work in the individual domains takes place at the project level. The project managers implement the selected projects autonomously and regularly report the project work's status to the program management.

The first draft of the masterplan comprised the overarching objectives and defined the framework for the planning and concretization of the SC initiative. We derived seven domains that impacted the current organizational structure: broadband, e-government, economy, mobility, living, infrastructure, and education. These domains have been used to create structures in several ways and play a crucial role in organizing the SC initiative. For instance, the participatory activities (incl. a series of public ideation workshops) were structured around the domains, each domain has one leader (e.g. a prominent businessperson or academic), and each project idea is classified and managed in accordance with the domains. This structure through conceptual subdivision supports the program and portfolio management of the whole initiative by creating an overview and making SC more concrete and understandable for all stakeholders.

The SC architecture goals included, amongst others, cost efficiency, uniformity, and data integrity. The architecture principles are divided into business-, data-, technology-, and application principles, and are supported by related architecture standards, governance, roles, and processes. The starting point for these was The Open Group Architecture Framework (TOGAF), since this framework is widely accepted by researchers and practitioners in the field. The masterplan also defined a comprehensive platform, which is an organizational and technological structure for SC. On the one hand, a platform has certain requirements, which limits the procurement and project development options, because it excludes offers or approaches that are not fit for the platform. Since establishing a platform can be very costly, it also requires an up-front investment. On the other hand, platforms serve to unite independent developers' distinctive expertise; even after their adoption, platforms can increase their value in a competitive market [37].

The innovation center supports research and prototype development. In practice, the center comprises various stakeholders who collaborate to research and test innovative technological or organizational solutions for the initiative, as well as providing the SC steering committee and working 
groups with advice, assess ideas, and evaluate project outcomes. Further, The SC education and qualification program began in the spring of 2019 and is, as far as we could determine, a unique professional training in Germany, especially in the context of an SC initiative.

Based on our observations of the SC initiative and discussions with the program management, we developed a responsibility assignment matrix (see table 1) that assigns roles for each governance process to the organizational structures. We use this matrix to assign accountabilities to structures, which means that it exhibits features similar to those of a RACI matrix widely accepted by practitioners [28]. The structure, which is accountable (A) for a task, can delegate it to others who are responsible (R) for executing it. Other structures are consulted (C) or informed (I).

\section{Discussion and conclusion}

We have described our ADR-guided development process and resulting SC governance regime. A central insight we gained during the process is that a 3-level control, including a strategic steering committee, operative program management, and individual PM is a crucial element for the efficient realization of the initiative's goals. Thus, PM and PPM, but also stakeholder communication and architecture management turned out to be key capabilities. Also, adapting an organizational culture to the requirements of an SC transformation revealed several tensions which were resolved in part through training and qualification.

The case city has certain characteristics that render this SC initiative fairly representative, including the city's size, as well as the organizational capacity, resource, critical mass, and infrastructure challenges it faces. Nonetheless, in further research it will be crucial to identify those context factors that vary in other SC initiatives when setting up their governance regime. One such factor is the necessary amount of effort regarding the creation of networks among the various stakeholders. In the case of Duisburg, the SC initiatives in the region are relatively isolated and the lack of an overarching - state-wide or federal - institution to systematically connect the potential stakeholders and efficiently create SC solutions required investing relatively much time and effort in stakeholder networking. Moreover, the lack of an overarching institution to provide frameworks and standards for the SC initiative required great efforts to establish the corresponding structures. Thus, in future research it would be valuable to investigate the effects of cross-city SC support activities on SC governance regimes.

Despite such context-dependent aspects, the resulting governance model evolved organically from the general challenges for SC initiatives in medium- sized cities, including the multi-layered authority structure and the large variety of stakeholders. As mentioned in section 2, existing SC literature provides little detail about the actual structures and processes involved in the governance of SC initiatives. Our detailed model, based on the requirements of a representative case as well as on seminal principles garnered from IS literature, thus provides a significant theoretical contribution to this field. Nevertheless, the results presented in this paper are subject to the obvious limitation of being based on experience from a single case. Given our participatory role as researchers in this initiative, it was a natural choice to concentrate our observations on this case. However, the generalizability of our results should in further research be assessed in respect of other SC initiatives. But despite this limitation, given the theoretical foundations and practical experiences from which our presented model has evolved, the latter may serve as valuable orientation for other SC initiatives in medium-sized cities.

\section{References}

[1] Alawadhi, S., A. Aldama-Nalda, H. Chourabi, et al., "Building Understanding of Smart City Initiatives", Electronic Government, Springer, Berlin, Heidelberg 2012, 40-53.

[2] Albino, V., U. Berardi, and R.M. Dangelico, "Smart Cities: Definitions, Dimensions, Performance, and Initiatives", Journal of Urban Technology 221, 2015, pp. 3-21.

[3] Anthopoulos, L., and P. Fitsilis, "Exploring architectural and organizational features in smart cities", 16th International Conference on Advanced Communication Technology, Global IT Research Institute (GIRI) 2014, 190-195.

[4] Bryson, J.M., "What to do when Stakeholders matter: Stakeholder Identification and Analysis Techniques", Public Management Review 61, 2004, pp. 21-53.

[5] Butt, A., M. Naaranoja, and J. Savolainen, "Project change stakeholder communication", International Journal of Project Management 348, 2016, pp. 1579-1595.

[6] Campbell, J., C. McDonald, and T. Sethibe, "Public and private sector IT governance: Identifying contextual differences", Australasian Journal of Information Systems 162, 2010.

[7] Caragliu, A., C. Del Bo, and P. Nijkamp, "Smart cities in Europe", Journal of urban technology 182, 2011, pp. 65-82.

[8] Castelnovo, W., G. Misuraca, and A. Savoldelli, "Smart Cities Governance: The Need for a Holistic Approach to Assessing Urban Participatory Policy Making", Social Science Computer Review 346, 2016, pp. 724-739.

[9] Chourabi, H., T. Nam, S. Walker, et al., "Understanding Smart Cities: An Integrative Framework", IEEE 2012, 22892297.

[10] Cocchia, A., "Smart and Digital City: A Systematic Literature Review", In R.P. Dameri and C. Rosenthal- 
Sabroux, eds., Smart City. Springer International Publishing, Berlin, Heidelberg, 2014, 13-43.

[11] Curwell, S., M. Deakin, I. Cooper, K. Paskaleva-Shapira, J. Ravetz, and D. Babicki, "Citizens' expectations of information cities: implications for urban planning and design", Building Research \& Information 331, 2005, pp. 5566.

[12] Daily, C.M., D.R. Dalton, and A.A. Cannella, "Corporate governance: Decades of dialogue and data", Academy of management review 283, 2003, pp. 371-382.

[13] Frost \& Sullivan, "Frost \& Sullivan Experts Announce Global Smart Cities to Raise a Market of Over \$2 Trillion by 2025", Frost \& Sullivan, 2018. https://ww2.frost.com/news/press-releases/frost-sullivanexperts-announce-global-smart-cities-raise-market-over-2trillion-2025/

[14] Giffinger, R., C. Fertner, H. Kramar, and E. Meijers, Cityranking of European Medium-Sized Cities, 2007.

[15] Hollands, R.G., "Will the real smart city please stand up?: Intelligent, progressive or entrepreneurial?", City 123, 2008, pp. 303-320.

[16] Kehua Su, J.L., "Smart City and the Applications", 2011.

[17] Kohler, T., "Corporate accelerators: Building bridges between corporations and startups", Business Horizons 593, 2016, pp. 347-357.

[18] Kunzmann, K.R., "Medium-Sized Towns, Strategic Planning and Creative Governance", In M. Cerreta, G. Concilio and V. Monno, eds., Making Strategies in Spatial Planning: Knowledge and Values. Springer Netherlands, Dordrecht, 2010, 27-45.

[19] Lederer, A.L., and V. Sethi, "The implementation of strategic information systems planning methodologies", MIS quarterly, 1988, pp. 445-461.

[20] Lombardi, P., S. Giordano, H. Farouh, and W. Yousef, "Modelling the smart city performance", Innovation: The European Journal of Social Sciences 252, 2012, pp. 137-149. [21] Maccani, G., N. Connolly, and B. Donnellan, "IT Governance in Smart Cities: An Exploratory Case Study of an European City Authority", Proceedings of the 27th European Conference on Information Systems (ECIS), 2019.

[22] Meijer, A., and M.P.R. Bolívar, "Governing the smart city: a review of the literature on smart urban governance", International Review of Administrative Sciences 822, 2016, pp. 392-408.

[23] Michalko, M., Thinkertoys: A Handbook of CreativeThinking Techniques, Ten Speed Press, 2006.

[24] Mullarkey, M.T., and A.R. Hevner, "An elaborated action design research process model", European Journal of Information Systems 281, 2019, pp. 6-20.

[25] Nam, T., and T.A. Pardo, "Conceptualizing Smart City with Dimensions of Technology, People, and Institutions", Proceedings of the 12th Annual International Digital Government Research Conference: Digital Government Innovation in Challenging Times, ACM 2011, 282-291.

[26] Ojo, A., E. Curry, and T. Janowski, "Designing next Generation Smart City Initiatives - Harnessing Findings and Lessons from a Study of Ten Smart City Programs", Tel Aviv, 2014, pp. 15.
[27] Organisation for Economic Cooperation and Development, "Population by region - Urban population by city size - OECD Data", theOECD, 2014. http://data.oecd.org/popregion/urban-population-by-citysize.htm

[28] PMI, A Guide to the Project Management Body of Knowledge (PMBOK Guide), Project Management Institute, Newtown Square, PA, 2008.

[29] Recker, J., Scientific Research in Information Systems - A Beginner's Guide, Springer, Berlin \& Heidelberg, Germany, 2013.

[30] Rodríguez Bolívar, M.P., "Smart Cities: Big Cities, Complex Governance?”, In M.P. Rodríguez-Bolívar, ed., Transforming City Governments for Successful Smart Cities. Springer International Publishing, Cham, 2015, 1-7.

[31] Schaffers, H., N. Komninos, M. Pallot, B. Trousse, M. Nilsson, and A. Oliveira, "Smart cities and the future internet: Towards cooperation frameworks for open innovation", The future internet assembly, Springer 2011, 431-446.

[32] Sein, M.K., O. Henfridsson, S. Purao, M. Rossi, and R. Lindgren, "Action Design Research", MIS Quarterly 351, 2011, pp. 37-56.

[33] Sepasgozar, S.M.E., S. Hawken, S. Sargolzaei, and M. Foroozanfa, "Implementing citizen centric technology in developing smart cities: A model for predicting the acceptance of urban technologies", Technological Forecasting and Social Change 142, 2019, pp. 105-116.

[34] Sethibe, T., J. Campbell, and C. McDonald, "IT governance in public and private sector organisations: examining the differences and defining future research directions", ACIS 2007 Proceedings, 2007, pp. 118.

[35] Susman, G.I., and R.D. Evered, "An Assessment of the Scientific Merits of Action Research", Administrative Science Quarterly 234, 1978, pp. 582.

[36] Tiemeyer, E., Handbuch IT-Management: Konzepte, Methoden, Lösungen und Arbeitshilfen für die Praxis, Carl Hanser Verlag GmbH Co KG, 2017.

[37] Tiwana, A., Platform ecosystems: aligning architecture, governance, and strategy, MK, Amsterdam; Waltham, MA, 2014.

[38] Van den Bergh, J., and S. Viaene, "Key Challenges for the Smart City: Turning Ambition into Reality", 2015 48th Hawaii International Conference on System Sciences, IEEE 2015, 2385-2394.

[39] Ward, J., and R. Elvin, “A new framework for managing IT-enabled business change", Information Systems Journal 93, 1999, pp. 197-221.

[40] Ward, J., P. Taylor, and P. Bond, "Evaluation and realisation of IS/IT benefits: an empirical study of current practice", European Journal of Information Systems 44, 1996, pp. 214-225.

[41] Washburn, D., U. Sindhu, S. Balaouras, R.A. Dines, N. Hayes, and L.E. Nelson, "Helping CIOs understand 'smart city' initiatives", Growth 172, 2009, pp. 1-17.

[42] Weill, P., and J.W. Ross, IT Governance: How Top Performers Manage IT Decision Rights for Superior Results, Harvard Business Review Press, Boston, 2004. 